\title{
When the Casimir energy is not a sum of zero-point energies
}

\author{
Luiz C. de Albuquerque* \\ Faculdade de Tecnologia de São Paulo-CEETEPS-UNESP, Praça Fernando Prestes, 30, 01124-060 São Paulo, SP, Brazil \\ R. M. Cavalcanti ${ }^{\dagger}$ \\ Instituto de Física, Universidade Federal do Rio de Janeiro, Caixa Postal 68528, 21945-970 Rio de Janeiro, RJ, Brazil
}

(Received 3 September 2001; published 15 January 2002)

\begin{abstract}
We compute the leading radiative correction to the Casimir force between two parallel plates in the $\lambda \Phi^{4}$ theory. Dirichlet and periodic boundary conditions are considered. A heuristic approach, in which the Casimir energy is computed as the sum of one-loop corrected zero-point energies, is shown to yield incorrect results, but we show how to amend it. The technique is then used in the case of periodic boundary conditions to construct a perturbative expansion which is free of infrared singularities in the massless limit. In this case we also compute the next-to-leading order radiative correction, which turns out to be proportional to $\lambda^{3 / 2}$.
\end{abstract}

DOI: 10.1103/PhysRevD.65.045004

PACS number(s): 11.10.Kk, 11.10.Gh, 12.20.Ds

\section{INTRODUCTION}

An important question in quantum field theory is the response of the vacuum fluctuations to perturbations of the space-time manifold: in the absence of a consistent quantum theory of gravitation in four space-time dimensions one is led to study vacuum fluctuations of matter or gauge fields in the presence of an external (i.e., classical) gravitational field [1]. One may also ask how the properties of a field theory are affected by the topology of space-time or by the presence of boundaries, which impose constraints on the fields. For instance, periodic boundary conditions on a spatial sector are a key ingredient in compactification schemes of Kaluza-Klein theories [2]. Boundary conditions (BC) are also used to describe complicated physical systems in a simplified mathematical framework. In the electromagnetic Casimir effect [3] one considers classical conductor plates (perfect mirrors), with the field satisfying Dirichlet BC on them. The analogous condition in the MIT bag model is the perfect confinement of quarks and gluons to the interior of hadrons [4]. In thermal field theory, periodic or antiperiodic BC in the imaginary-time are the starting point of the Matsubara formalism [5]. Finally, the study of surface effects on the critical properties of a (magnetic, binary liquid, etc.) system leads in many cases to the analysis of scalar field theories subject to certain boundary conditions [6].

Although $\mathrm{BC}$ have been extensively studied in quantum field theory models, there remains a lot of questions to be answered. In this paper we will investigate some unusual features of periodic and Dirichlet BC on one spatial coordinate. In the remainder of the Introduction we will give some motivations to the study of these particular types of boundary conditions.

Quantum field theories in compactified spaces (i.e., with periodic boundary conditions in some spatial directions) have been the subject of considerable interest in the literature [7-9]. The calculation of the effective potential in spontane-

\footnotetext{
*Email address: 1claudio@fatecsp.br

†Email address: rmoritz@if.ufrj.br
}

ously broken symmetry theories shows that the compactification process may introduce a mechanism for dynamical symmetry restoration. Generation of a dynamical mass is connected with the inclusion of a new scale, the compactification radius $R$.

There is a complete mathematical analogy between compactified field theory and thermal field theory (TFT) in the Matsubara formalism. In the latter, the inverse temperature $\beta=1 / T$ is the compactification radius in the imaginary-time direction. The well-known fact that thermal effects do not lead to new ultraviolet divergences in TFT (besides the usual ones found at $T=0$ ) [5] applies as well to compactified field theories. On the other hand, the infrared properties of the TFT are very different from the ones at zero temperature. The free energy of massless $\lambda \Phi^{4}$ theory in three spatial dimensions develops new infrared divergences at order $\lambda^{2}$ in perturbation theory [5]. The dominant infrared divergences come from the $n=0$ mode of the loop momenta. A proper treatment of the collective effects leads to a correction of order $\lambda^{3 / 2}$ to the free energy.

The infrared behavior of the compactified field theory mimics the one at finite temperature, at least in the case of one spatial compactified coordinate. In a perturbative treatment, the $n=0$ mode generates new infrared divergences in the compactified version of the $\lambda \Phi^{4}$ theory. This seems to be not so well-appreciated in the literature. To fill this gap, we apply the resummation method developed by Braaten, Pisarski and others (in the context of TFT) [10-12] to the compactified $\lambda \Phi^{4}$ theory.

Symmetries in quantum field theory put very stringent conditions on the perturbative renormalization of a model and in its physical predictions. Lorentz invariance (rotational invariance in the Euclidean case) is of paramount importance in this respect. However, external conditions or dynamical effects may lead to its breakdown. There is a growing interest on effective field theories in which this occurs (e.g., noncommutative field theories [13], anisotropic systems [14], and Chern-Simons theories [15]). Theories defined in finite volumes or in the presence of macroscopic bodies (as in the Casimir effect) may provide useful insights on the conse- 
quences of lack of Lorentz symmetry to the renormalization program.

Recently, there has been much effort in the computation of radiative corrections to the Casimir energy, specially in QED [16]. One of the purposes of this paper is to discuss an alternative method to compute such corrections. For simplicity we work with the $\lambda \Phi^{4}$ theory subject to Dirichlet boundary conditions on a pair of parallel plates. The method is based on a resummation of the perturbative series for the two-point Green function, and leads to a Klein-Gordon equation in which the one-loop self-energy acts as an effective scalar potential. In four space-time dimensions this equation can be solved exactly in the massless case. The new set of (resummed) eigenvalues contain radiative corrections of all orders in $\lambda$, and reduce to the free ones for $\lambda=0$. The computation of the sum of effective zero-point energies, including non-perturbative corrections and renormalization issues, is discussed in detail.

The plan of the paper is as follows. In Sec. II we fix the conventions and discuss the resummation technique in the $\lambda \Phi^{4}$ theory with Dirichlet boundary conditions. We solve the effective Klein-Gordon equation, and obtain the "improved" eigenvalues. The solution is used to obtain the resummed Casimir energy, including radiative corrections. In Sec. III we discuss the resummation of the vacuum energy in the case of periodic boundary conditions; this sheds some light on the results of Sec. II. In the Conclusion we discuss the drawbacks of this method as well as other minor points. Finally, three Appendixes collect some mathematical results used in the paper.

\section{DIRICHLET BOUNDARY CONDITIONS}

Boundary conditions breaking the full Lorentz invariance in general pose new problems to the renormalization program. For some geometries and boundary conditions (depending also on the spin of the field) it may be necessary to introduce surface counterterms besides the bulk ones. For instance, in the MIT bag model the free energy is ill defined at one-loop due to an extra singularity which shows up as the surface is approached [17]. The standard recipe associates a free parameter to each distinct singular term, included as a new counterterm in the starting Lagrangian. If this procedure continues to all orders, with the consequent loss of predictive power, we say that the theory is non-renormalizable due to the boundary conditions.

In a remarkable paper, Symanzik gave strong arguments showing the renormalizability of the $\Phi^{4}$ theory in the presence of flat boundaries [18]. In particular, he showed that the renormalized Casimir pressure for disjoint boundaries and Dirichlet $\mathrm{BC}$ is finite to all orders in perturbation theory. He also verified explicitly that no surface counterterms are needed in the computation of the two-loop vacuum energy. $\Phi^{4}$-type theories are still renormalizable for more general boundary conditions and surfaces, but at the price of introducing surface counterterms $[6,18]$. We wish to point out here that many proposals have been made in order to avoid the surface-like singularities. These include, among others, the "softening" of the Dirichlet BC $[19,20]$ or treating the boundaries as quantum mechanical objects with a nonzero position uncertainty [21]. However, this question is outside the scope of our present discussion.

A key ingredient in our computation of the Casimir energy is the self-energy of the field, as it determines the shift in the single-particle energy levels. Since there is some disagreement among existent results in the literature $[7,8,18,25]$, we present its computation in some detail.

\section{A. Self-energy}

We work in $D=(d+1)+1$ dimensional Minkowski space-time, and define $x^{\mu} \equiv(t, \mathbf{x}, z)$, with $\mathbf{x}=\left(x^{1}, \ldots, x^{d}\right)$. The renormalized Lagrangian reads $\left[\hbar=c=1, \eta_{\mu \nu}=\right.$ diag $(+,-, \ldots,-)]$

$$
\mathcal{L}=\mathcal{L}_{0}+\mathcal{L}_{I}=\left\{\frac{1}{2}(\partial \Phi)^{2}-\frac{1}{2} m^{2} \Phi^{2}\right\}+\left\{-\frac{\lambda}{4 !} \Phi^{4}+\mathcal{L}_{\mathrm{ct}}\right\},
$$

with $\mathcal{L}_{\text {ct }}$ the counterterm Lagrangian.

We impose Dirichlet $\mathrm{BC}$ on a pair of plates at $z=0$ and $z=l: \quad \Phi(z=0)=\Phi(z=l)=0$. The bare Feynman propagator with Dirichlet BC may be written as an expansion in multiple reflections [22]:

$$
\Delta_{F}\left(x, x^{\prime}\right)=\sum_{n=-\infty}^{\infty}\left[\Delta_{F}^{(0)}\left(x_{n}-x_{+}^{\prime}\right)-\Delta_{F}^{(0)}\left(x_{n}-x_{-}^{\prime}\right)\right],
$$

where $x_{n}=(t, \mathbf{x}, z+2 n l), x_{ \pm}^{\prime}=\left(t^{\prime}, \mathbf{x}^{\prime}, \pm z^{\prime}\right)$, and $\Delta_{F}^{(0)}$ is the bulk free propagator, which for $D>2$ is given by [23]

$\Delta_{F}^{(0)}(x)$

$$
=\frac{1}{(2 \pi)^{D / 2}}\left(\frac{m}{\sqrt{-x^{2}+i \epsilon}}\right)^{(D-2) / 2} K_{(D-2) / 2}\left(m \sqrt{-x^{2}+i \epsilon}\right),
$$

with $\sqrt{-x^{2}+i \epsilon}=i \sqrt{x^{2}}$ if $x^{2}>0 \quad\left(\epsilon \rightarrow 0^{+}\right)$. Actually, what we are interested in is $\Delta_{F}(x, x)$. It follows from Eq. (2) that

$$
\Delta_{F}(x, x)=\sum_{n=-\infty}^{\infty}\left[\Delta_{F}^{(0)}(2 n l)-\Delta_{F}^{(0)}(2 z+2 n l)\right] .
$$

The term $\Delta_{F}^{(0)}(0)$ contains the usual UV singularity. It can be removed, as usual, by a mass renormalization.

In the massless case the bulk free propagator gets simplified, and it is possible to find a closed expression for $\Delta(x)$ $\equiv \Delta_{F}(x, x)-\Delta_{F}^{(0)}(0)$. Using

$$
\Delta_{F}^{(0)}(x ; m=0)=\frac{\Gamma\left(\frac{D}{2}-1\right)}{4 \pi^{D / 2}}|x|^{2-D},
$$

one finds, for $D=4$, 


$$
\Delta(x ; m=0)=\frac{1}{16 \pi^{2} l^{2}}\left[2 \psi^{\prime}(1)-\psi^{\prime}\left(\frac{z}{l}\right)-\psi^{\prime}\left(1-\frac{z}{l}\right)\right]
$$

where $\psi(x)$ is the digamma function [24]. Equation (6) can be simplified to

$$
\Delta(x ; m=0)=-\frac{1}{16 l^{2}}\left[\csc ^{2}\left(\frac{\pi z}{l}\right)-\frac{1}{3}\right] \quad(D=4) .
$$

Let us take a closer look at the $D=3$ case, keeping $m$ $\neq 0$. We obtain from Eqs. (3) and (4), after changing the summation variables and using the explicit form of $K_{1 / 2}(x)$,

$$
\begin{aligned}
\Delta(x)= & \frac{1}{8 \pi l}\left[2 e^{-2 m l} S(2 m l, 1)-e^{-2 m z} S\left(2 m l, \frac{z}{l}\right)\right. \\
& \left.-e^{-2 m(l-z)} S\left(2 m l, 1-\frac{z}{l}\right)\right],
\end{aligned}
$$

where

$$
S(a, b) \equiv \sum_{n=0}^{\infty} \frac{e^{-a n}}{n+b}
$$

The massless limit must be taken with care, as each of the series in Eq. (8) is logarithmically divergent. As we shall show, the divergent terms cancel in the complete formula (8). Indeed, the asymptotic limit of $S(2 m l, b)$ as $m \rightarrow 0$ is given by

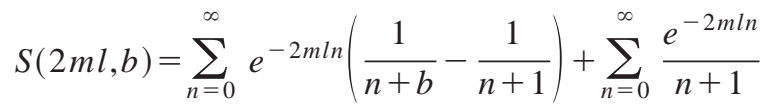

$$
\begin{aligned}
& m \rightarrow 0 \\
& \sim-\gamma-\psi(b)-\ln (2 m l)+O(m l),
\end{aligned}
$$

where $\gamma=0.577 \ldots$ is the Euler constant. The logarithmic terms cancel in Eq. (8), so that we can now take the limit $m \rightarrow 0$ safely, obtaining

$$
\Delta(x ; m=0)=\frac{1}{8 \pi l}\left[2 \gamma+\psi\left(\frac{z}{l}\right)+\psi\left(1-\frac{z}{l}\right)\right] \quad(D=3) .
$$

The renormalized one-loop self-energy is given by $\Sigma^{(1)}(x)=(\lambda / 2) \Delta_{F}(x, x)+\delta m^{2}$. The mass counterterm is fixed by the condition $\lim _{l \rightarrow \infty} \Sigma^{(1)}=0$. This amounts to remove the contribution of the bulk free propagator from Eq. (4). With this choice of mass renormalization we have $\Sigma^{(1)}(x)=(\lambda / 2) \Delta(x)$. Therefore, the self-energy is infrared finite in the massless case also at $D=3$, in disagreement with Ref. [25]. [However, it is infrared divergent in the case of Neumann boundary conditions. The propagator is then given by Eq. (2) with the minus sign on its right-hand side (RHS) replaced by a plus sign. As a consequence, the logarithmic terms in the massless limit of $\Delta(x)$ do not cancel.]
From now on, we shall focus our attention on the massless case at $D=4$.

\section{B. Radiative corrections to the Casimir energy: A heuristic approach}

Computations of the Casimir energy in the literature are restricted to perturbation theory. A non-perturbative calculation would be a very interesting result. Our goal here is more modest. We will discuss the computation of the Casimir energy in the approximation where the two-point Green function is dressed with an arbitrary number of insertions of the one-loop self-energy ("daisy" resummation). This approximation contains the leading correction in a $1 / N$ expansion. (In our case, however, $N=1$. With this caveat in mind, let us proceed.) The Casimir energy will be given formally by

$$
E=\frac{1}{2} \sum_{\alpha} \omega_{\alpha}
$$

where $\omega_{\alpha}$ are the positive poles of the dressed two-point function $\widetilde{G}^{(2)}$ in the frequency domain.

To compute $\widetilde{G}^{(2)}$ we note that it satisfies

$$
\left[\partial_{x}^{2}+\Sigma^{(1)}(x)\right] \widetilde{G}^{(2)}\left(x, x^{\prime}\right)=-i \delta^{(4)}\left(x, x^{\prime}\right) .
$$

As usual, the solution to Eq. (13) can be written as

$$
\widetilde{G}^{(2)}\left(x, x^{\prime}\right)=-i \sum_{\alpha} \frac{\phi_{\alpha}(x) \phi_{\alpha}^{*}\left(x^{\prime}\right)}{\Lambda_{\alpha}}
$$

where $\Lambda_{\alpha}$ and $\phi_{\alpha}(x)$ are the eigenvalues and (normalized) eigenfunctions, respectively, of the Klein-Gordon operator $\partial^{2}+\Sigma^{(1)}$ :

$$
\left[\partial^{2}+\Sigma^{(1)}(x)\right] \phi_{\alpha}(x)=\Lambda_{\alpha} \phi_{\alpha}(x) .
$$

Since $\Sigma^{(1)}(x)$ is a function of $z$ alone, we can reduce the above equation to an ordinary differential equation by writing $\phi(x)=e^{-i \omega t+i \mathbf{p} \cdot \mathbf{x}} \varphi(z)$ :

$$
\left\{-\frac{d^{2}}{d z^{2}}-\sigma^{2}-\frac{\pi^{2} g}{l^{2}}\left[\csc ^{2}\left(\frac{\pi z}{l}\right)-\frac{1}{3}\right]\right\} \varphi(z)=0,
$$

where $\sigma^{2} \equiv \Lambda+\omega^{2}-\mathbf{p}^{2}$ and $g \equiv \lambda / 32 \pi^{2}$. Now we make the change of variable $z=l y / \pi$ and get

$$
\left(\frac{d^{2}}{d y^{2}}+k^{2}+\frac{g}{\sin ^{2} y}\right) \varphi(y)=0 \quad\left(k^{2} \equiv \frac{l^{2} \sigma^{2}}{\pi^{2}}-\frac{g}{3}\right) .
$$

Equation (17) may be viewed as the time-independent Schrödinger equation for a particle of mass $\tilde{m}=1 / 2$ moving in the potential $V(y)=-g \csc ^{2} y$ (inverted Poschl-Teller), with energy $E=k^{2}$. Its solution is discussed in Appendix A. In particular, it is shown that $k^{2}=(n+s)^{2} \quad(n=1,2, \ldots)$, with $s \equiv \frac{1}{2}(-1+\sqrt{1-4 g})$. From the definition of $k^{2}$ and $\sigma^{2}$ it follows that the eigenvalues of the Klein-Gordon operator have the form 


$$
\Lambda=-\omega^{2}+\mathbf{p}^{2}+\frac{\pi^{2}}{l^{2}}\left[(n+s)^{2}+\frac{g}{3}\right] \quad(n=1,2, \ldots) .
$$

From Eqs. (14) and (18) it follows that the (positive) poles of $\widetilde{G}^{(2)}$ are given by

$$
\omega_{n}(\mathbf{p})=\sqrt{\mathbf{p}^{2}+\frac{\pi^{2}}{l^{2}}\left[(n+s)^{2}+\frac{g}{3}\right]} \quad(n=1,2, \ldots) .
$$

Before we proceed with the calculation of the Casimir energy, a remark is in order here. As we have seen, the (renormalized) one-loop self-energy is a function of $x$. It may be tempting to interpret $\Sigma^{(1)}(x)$ (more generally, $m^{2}$ $\left.+\Sigma^{(1)}(x)\right)$ as a position-dependent (squared) mass of the field. A problem would then occur in regions where $\Sigma^{(1)}(x)<0$, for this could imply the presence of tachyons in the theory. For that reason, Ford and Yoshimura [7] argued that models which exhibits this behavior (such as the one we are considering) are unphysical. However, the analysis of Eq. (16), summarized in Appendix A, shows that its solutions do not have imaginary frequencies as long as $\lambda<\lambda_{\text {crit }}=8 \pi^{2}$ (which is anyway well outside the range of validity of perturbation theory). The one-loop effective theory is consistent in this case. On the other hand, for $\lambda>8 \pi^{2}$ the Schrödinger equation (16) leads to an energy spectrum which is unbounded from below, rendering the associated effective field theory ill-defined. This solves a long-standing problem of interpretation.

Substituting the eigenfrequencies (19) into Eq. (12) we obtain the following expression for the Casimir energy per unit area:

$$
\mathcal{E}=\left.\frac{1}{2} \mu^{1-2 \nu} \sum_{n=1}^{\infty} \int \frac{d^{2} p}{(2 \pi)^{2}}\left\{\mathbf{p}^{2}+\frac{\pi^{2}}{l^{2}}\left[(n+s)^{2}+\frac{g}{3}\right]\right\}\right|_{\nu=1 / 2} ^{\nu} .
$$

The formal sum over zero-point energies has been analytically regularized; we shall set $\nu=1 / 2$ at the end of the calculation. The factor $\mu^{1-2 \nu}$, where $\mu$ is a mass parameter, keeps the RHS of Eq. (20) with the dimension of energy per unit area.

Integrating Eq. (20) over p, we get

$$
\mathcal{E}=\frac{\mu^{1-2 \nu}}{8 \pi} \frac{\Gamma(-\nu-1)}{\Gamma(-\nu)}\left(\frac{\pi}{l}\right)^{2(\nu+1)} \mathcal{H}\left(-\nu-1 ; s, \frac{g}{3}\right),
$$

where the function $\mathcal{H}\left(z ; s, a^{2}\right)$ is defined as

$$
\mathcal{H}\left(z ; s, a^{2}\right) \equiv \sum_{n=1}^{\infty}\left[(n+s)^{2}+a^{2}\right]^{-z}
$$

The series converges for $\mathfrak{R} z>1 / 2$. The analytical continuation of $\mathcal{H}\left(z ; s, a^{2}\right)$ to the whole complex $z$ plane is performed in Appendix B. Substituting the result into Eq. (21) we obtain [26]

$$
\begin{aligned}
\mathcal{E}= & \frac{\mu^{1-2 \nu}}{8 \pi} \frac{\Gamma(-\nu-1)}{\Gamma(-\nu)}\left(\frac{\pi}{l}\right)^{2(\nu+1)}\left\{\frac{1}{2}\left[(1+s)^{2}+\frac{g}{3}\right]^{\nu+1}\right. \\
& +i \int_{0}^{\infty} d t \frac{f_{\nu}(1+i t)-f_{\nu}(1-i t)}{e^{2 \pi t}-1}-\frac{(1+s)^{2 \nu+3}}{2 \nu+3} \\
& \left.\times F\left(-\nu-1,-\nu-\frac{3}{2} ;-\nu-\frac{1}{2} ;-\frac{g}{3(1+s)^{2}}\right)\right\},
\end{aligned}
$$

where $f_{\nu}(x) \equiv\left[(x+s)^{2}+g / 3\right]^{\nu+1}$ and $F(\alpha, \beta ; \gamma ; z)$ is the hypergeometric function. From the definition of the latter it follows that $\mathcal{E}$ has a simple pole at $\nu=1 / 2$ (in fact, it has poles at $\nu=-3 / 2,-1 / 2,1 / 2,3 / 2, \ldots)$. This requires that $\mathcal{E}$ be renormalized before we set $\nu=1 / 2$. In general, this is done by subtracting from $\mathcal{E}$ its value at $l \rightarrow \infty$. Unfortunately, such a prescription does not work in the present case, since, according to Eq. (23), the Casimir energy per unit area has the form $\mathcal{E}=C(\nu) / l^{2(\nu+1)}$.

One can obtain a hint on what is going wrong by noting that the residue of $\mathcal{E}$ at $\nu=1 / 2$ is of second order in $g$ (or $\lambda$ ). This is consistent with the fact that we have worked with the one-loop two-point Green function, which is (formally) correct only to first order in the coupling constant. Since the $\lambda \Phi^{4}$ theory is perturbatively renormalizable in $D=4$, one may suspect that in order to obtain a finite (or at least renormalizable) $\mathcal{E}$ to order $\lambda^{n}$ one must work within an approximation in which the two-point Green function is dressed with the $n$-loop self-energy. As we show below, this is not sufficient or necessary. In spite of that, the argument suggests that Eq. (23) cannot be trusted beyond order $\lambda$.

Expanding the RHS of Eq. (23) in a power series in $\lambda$ and making $\nu \rightarrow 1 / 2$, we obtain

$$
\mathcal{E}=\frac{1}{l^{3}}\left[-\frac{\pi^{2}}{1440}+\frac{\lambda}{9216}+\cdots\right]
$$

The first term is the usual free Casimir energy (per unit area). The second term is the leading radiative correction to it. It overestimates the correct result [18] by a factor of 2 . This discrepancy occurs because the method we have used to compute the Casimir energy only works in the absence of interactions. To show this, we start by noting that one can define the Casimir energy as

$$
E=\int d^{D-1} x\left\langle 0\left|T_{00}(x)\right| 0\right\rangle,
$$

where $T_{\mu \nu}$ is the energy-momentum tensor. In the case we are considering (the massless $\lambda \Phi^{4}$ theory in $D=4$ ), we have

$$
T_{00}=\frac{1}{2}\left(\partial_{0} \Phi\right)^{2}+\frac{1}{2}(\vec{\nabla} \Phi)^{2}+\frac{\lambda}{4 !} \Phi^{4}
$$

Moving the differential operators outside the brackets, we can rewrite the vacuum expectation value of $T_{00}$ in terms of $n$-point Green functions $G^{(n)}$ : 


$$
\begin{aligned}
\left\langle 0\left|T_{00}(x)\right| 0\right\rangle= & \lim _{x^{\prime} \rightarrow x} \frac{1}{2}\left(\partial_{0} \partial_{0}^{\prime}+\vec{\nabla} \cdot \vec{\nabla}^{\prime}\right) G^{(2)}\left(x, x^{\prime}\right) \\
& +\frac{\lambda}{4 !} G^{(4)}(x, \ldots, x) .
\end{aligned}
$$

On the other hand, using Eq. (13) and the spectral representation of $\widetilde{G}^{(2)}$, Eq. (14), one can easily show that

$$
\begin{aligned}
\frac{1}{2} \sum_{\alpha} \omega_{\alpha}= & \int d^{D-1} x \lim _{x^{\prime} \rightarrow x} \frac{1}{2}\left[\partial_{0} \partial_{0}^{\prime}+\vec{\nabla} \cdot \vec{\nabla}^{\prime}\right. \\
& \left.+\Sigma^{(1)}(x)\right] \widetilde{G}^{(2)}\left(x, x^{\prime}\right) .
\end{aligned}
$$

It follows that the sum of (one-loop) zero-point energies differs from the true vacuum energy by

$$
\begin{aligned}
\Delta E= & \int d^{D-1} x\left[\lim _{x^{\prime} \rightarrow x} \frac{1}{2}\left(\partial_{0} \partial_{0}^{\prime}+\vec{\nabla} \cdot \vec{\nabla}^{\prime}\right) \Delta G^{(2)}\left(x, x^{\prime}\right)\right. \\
& \left.-\frac{1}{2} \Sigma^{(1)}(x) \widetilde{G}^{(2)}(x, x)+\frac{\lambda}{4 !} G^{(4)}(x, \ldots, x)\right],
\end{aligned}
$$

where $\Delta G^{(2)} \equiv G^{(2)}-\widetilde{G}^{(2)}$. While the first line of Eq. (29) is formally $O\left(\lambda^{2}\right)$, the second one is $O(\lambda)$. This explains why the second term in Eq. (24) is incorrect.

It is important to note that Eqs. (12) and (26) would lead to distinct results even if we had worked with the complete two-point Green function. The difference between them would then be given by

$$
\begin{aligned}
\Delta E= & \int d^{D-1} x\left\{-\frac{1}{2} \int d^{D} y \Sigma(x, y) G^{(2)}(y, x)\right. \\
& \left.+\frac{\lambda}{4 !} G^{(4)}(x, \ldots, x)\right\} .
\end{aligned}
$$

A perturbative evaluation of the above expression shows that $\Delta E$ would still be of order $\lambda$. Physically, this discrepancy is due to the fact that, in contrast with the free theory, the interacting theory is not equivalent to a collection of independent harmonic oscillators. The sum of zero-point energies, Eq. (12), takes into account only the Lamb shift on the single-particle energy levels caused by the interaction; the difference $\Delta E$ accounts for the residual interaction among the (anharmonic) oscillators.

The above discussion also shows that the Casimir energy is not determined solely by the two-point Green function, but also (in the $\lambda \Phi^{4}$ theory) by the four-point function. In particular, in order to consistently remove the $O\left(\lambda^{2}\right) \mathrm{UV}$ singularity in Eq. (23) one must not only obtain $G^{(2)}$ to that order, but also $G^{(4)}$ to $O(\lambda)$. These ideas will be illustrated in the next section in the simpler case of periodic boundary conditions.

\section{PERIODIC BOUNDARY CONDITIONS}

\section{A. Conventional perturbation theory}

The free Feynman propagator for the field $\Phi$ obeying periodic boundary conditions in the $z$-direction, $\Phi(t, \mathbf{x}, z$ $+R)=\Phi(t, \mathbf{x}, z)$, is given by

$$
\Delta_{F}\left(x, x^{\prime}\right)=\frac{i}{R} \int \frac{d \omega}{2 \pi} \sum_{n=-\infty}^{\infty} \int \frac{d^{d} p}{(2 \pi)^{d}} \frac{e^{-i p^{\mu}\left(x_{\mu}-x_{\mu}^{\prime}\right)}}{\omega^{2}-\mathbf{p}^{2}-q_{n}^{2}-m^{2}+i \epsilon}=\frac{1}{2 R} \sum_{n=-\infty}^{\infty} \int \frac{d^{d} p}{(2 \pi)^{d}} \frac{e^{-i \omega_{n}(\mathbf{p})\left|t-t^{\prime}\right|+i \mathbf{p} \cdot\left(\mathbf{x}-\mathbf{x}^{\prime}\right)+i q_{n}\left(z-z^{\prime}\right)}}{\omega_{n}(\mathbf{p})},
$$

where $\quad p^{\mu}=\left(\omega, \mathbf{p}, q_{n}\right), q_{n}=2 \pi n / R, \quad$ and $\quad \omega_{n}(\mathbf{p})$ $\equiv \sqrt{\mathbf{p}^{2}+q_{n}^{2}+m^{2}}$. Since $\Delta_{F}\left(x, x^{\prime}\right)=\Delta_{F}\left(x-x^{\prime}\right)$, such boundary conditions do not break translational invariance.

The renormalized vacuum energy density may be computed from Eqs. (25)-(27), but its perturbative expansion is more easily derived from the vacuum persistence amplitude:

$$
\varepsilon=\lim _{T \rightarrow \infty} \frac{i}{V T} \ln \left[\int \mathcal{D} \Phi \exp \left(i \int d^{D} x \mathcal{L}\right)\right]+\Lambda
$$

The last term in Eq. (32) is fixed by the renormalization condition $\lim _{R \rightarrow \infty} \varepsilon(x)=0$. Due to the translational invariance the vacuum energy density does not depend on $x$. (A remark on notation: $\varepsilon$ denotes the Casimir energy per unit volume, while $\mathcal{E}$ denotes the Casimir energy per unit area. They are related, in the case of periodic BC, by $\varepsilon=\mathcal{E} / R$.)
To first order in $\lambda$, we obtain from Eq. (32) the wellknown results $\left[\varepsilon=\varepsilon^{(0)}+\varepsilon^{(1)}+\cdots, \varepsilon^{(n)}=O\left(\lambda^{n}\right)\right]$

$$
\begin{aligned}
& \varepsilon^{(0)}=\frac{1}{2 R} \sum_{n=-\infty}^{\infty} \int \frac{d^{d} p}{(2 \pi)^{d}} \omega_{n}(\mathbf{p})+\Lambda^{(0)}, \\
& \varepsilon^{(1)}=\frac{\lambda}{8}\left[\Delta_{F}(0)\right]^{2}+\frac{1}{2} \delta m^{2} \Delta_{F}(0)+\Lambda^{(1)},
\end{aligned}
$$

where $\delta m^{2}$ is the one-loop mass counterterm. The one-loop self-energy is given by

$$
\Sigma^{(1)}=\frac{\lambda}{2} \Delta_{F}(0)+\delta m^{2} .
$$


In order to compute the quantities above, it is convenient to define the function

$$
\Psi_{\epsilon}(\alpha) \equiv \frac{\mu^{\epsilon}}{2 R} \sum_{n=-\infty}^{\infty} \int \frac{d^{d-\epsilon} p}{(2 \pi)^{d-\epsilon}}\left(\mathbf{p}^{2}+q_{n}^{2}+m^{2}\right)^{\alpha}
$$

where $\mu$ is an arbitrary mass scale. We then have $\Delta_{F}(0)$ $=\lim _{\epsilon \rightarrow 0} \Psi_{\epsilon}(-1 / 2)$ and $\varepsilon^{(0)}=\lim _{\epsilon \rightarrow 0}\left[\Psi_{\epsilon}(1 / 2)+\Lambda^{(0)}\right]$.

The computation of $\Psi_{\epsilon}$ is discussed in Appendix C. There we show that $\Psi_{\epsilon}$ may be written (in the limit $\epsilon \rightarrow 0$ ) as the sum of two terms, namely

$$
\lim _{\epsilon \rightarrow 0} \Psi_{\epsilon}(\alpha)=\mathcal{A}(\alpha)+\mathcal{B}(\alpha),
$$

where $\mathcal{A}(\alpha)$ and $\mathcal{B}(\alpha)$ are given by Eqs. (C8) and (C9), respectively. Only $\mathcal{A}(\alpha)$ depends on $R$, and vanishes when $R \rightarrow \infty$.

Before computing $\varepsilon^{(0)}$ and $\varepsilon^{(1)}$ we give our renormalization conditions. To first order in $\lambda$ two conditions are required. We fix $\Lambda$ and $\delta m^{2}$ by the conditions $\lim _{R \rightarrow \infty} \varepsilon(R)$ $=0$ and $\lim _{R \rightarrow \infty} \Sigma^{(1)}(R)=0$, respectively. This gives $\Lambda^{(0)}$ $=-\mathcal{B}(1 / 2), \quad \Lambda^{(1)}=(\lambda / 8)[\mathcal{B}(-1 / 2)]^{2}, \quad$ and $\quad \delta m^{2}=$ $-(\lambda / 2) \mathcal{B}(-1 / 2)$. It follows that $(\epsilon \rightarrow 0)$

$$
\begin{aligned}
\varepsilon^{(0)}(R) & =\mathcal{A}(1 / 2)=-\frac{2}{(2 \pi)^{D / 2}}\left(\frac{m}{R}\right)^{D / 2} F\left(\frac{D}{2} ; m R\right), \\
\varepsilon^{(1)}(R) & =\frac{\lambda}{8}[\mathcal{A}(-1 / 2)]^{2} \\
& =\frac{\lambda}{2(2 \pi)^{D}}\left(\frac{m}{R}\right)^{D-2}\left[F\left(\frac{D}{2}-1 ; m R\right)\right]^{2}
\end{aligned}
$$

where $F(s ; a)$ is defined in Eq. (C5). Taking $D=4$ and expanding in powers of $m R$ [Eqs. (C6) and (C7)] we thus obtain

$$
\begin{aligned}
\varepsilon= & \frac{1}{R^{4}}\left\{\left[-\frac{\pi^{2}}{90}+\frac{(m R)^{2}}{24}-\frac{(m R)^{3}}{12 \pi}+\cdots\right]\right. \\
& \left.+\lambda\left[\frac{1}{1152}-\frac{m R}{192 \pi}+\cdots\right]\right\}+\cdots
\end{aligned}
$$

Analogously, we obtain for the one-loop self-energy

$$
\begin{aligned}
\Sigma^{(1)} & =\frac{\lambda}{(2 \pi)^{D / 2}}\left(\frac{m}{R}\right)^{(D-2) / 2} F\left(\frac{D}{2}-1 ; m R\right) \\
& =\frac{\lambda}{R^{2}}\left[\frac{1}{24}-\frac{m R}{8 \pi}+\frac{(m R)^{2}}{16 \pi^{2}} \ln (m R)+\cdots\right] \quad(D=4) .
\end{aligned}
$$

The first term does not depend on $m$, and is sometimes called "topological mass" (squared). For reasons discussed in [1], we prefer the name "compactification mass" for $M$ $\equiv\left(\lambda / 24 R^{2}\right)^{1 / 2}$.

\section{B. Resummed perturbation theory}

From now on, let us focus the discussion on the massless theory $(m=0)$ in $D=4$. In this case, the second and higher order terms in the perturbative expansion of $\varepsilon$ are plagued with IR divergences. A qualitative analysis shows that the most IR divergent diagrams are the "ring" (or "daisy") ones. (These are just the diagrams with the greatest number of insertions of the one-loop self-energy in each order of perturbation theory.) As in the case of TFT [5], it is possible to sum these diagrams to all orders. The result is finite in the IR and is nonanalytic in $\lambda$, as we show below.

To avoid overcounting of diagrams in higher order calculations, it is convenient to redefine the free and the interacting parts of the Lagrangian by adding and subtracting to it the compactification mass term $\frac{1}{2} M^{2} \Phi^{2}[10-12]$ :

$$
\begin{aligned}
\mathcal{L}= & \widetilde{\mathcal{L}}_{0}+\widetilde{\mathcal{L}}_{I}=\left\{\frac{1}{2}(\partial \Phi)^{2}-\frac{1}{2} M^{2} \Phi^{2}\right\} \\
& +\left\{-\frac{\lambda}{4 !} \Phi^{4}+\frac{1}{2} M^{2} \Phi^{2}+\mathcal{L}_{\mathrm{ct}}\right\} .
\end{aligned}
$$

The free propagator (in momentum space) is now given by

$$
\widetilde{\Delta}_{F}(p)=\frac{i}{p^{2}-M^{2}+i \epsilon} .
$$

It coincides with the propagator of the original theory in the daisy approximation.

We remark that in a loop expansion of the vacuum energy (or of any other quantity) each insertion of the mass term in $\widetilde{\mathcal{L}}_{I}$ is to be formally counted as one loop, like the mass counterterm-otherwise taking $\widetilde{\mathcal{L}}_{0}$ as the new free Lagrangian would not cure the IR divergence problem [27].

The one-loop approximation to the vacuum energy is given by

$$
\widetilde{\varepsilon}^{(1)}=\frac{1}{2 R} \sum_{n=-\infty}^{\infty} \int \frac{d^{2} p}{(2 \pi)^{2}}\left(\mathbf{p}^{2}+q_{n}^{2}+M^{2}\right)^{1 / 2} .
$$

Using the results of Sec. III A and of Appendix C we obtain

$$
\begin{aligned}
\widetilde{\varepsilon}^{(1)}(R)= & -\frac{M^{2}}{2 \pi^{2} R^{2}} F(2 ; M R) \\
& +\lim _{\epsilon \rightarrow 0} \frac{\mu^{\epsilon} M^{4-\epsilon} \pi^{(3-\epsilon) / 2}}{\Gamma\left(-2+\frac{\epsilon}{2}\right)} \\
= & \frac{1}{R^{4}}\left[-\frac{\pi^{2}}{90}+\frac{\lambda}{576}-\frac{\lambda^{3 / 2}}{288 \sqrt{6} \pi}+O\left(\frac{\lambda^{2}}{\epsilon}\right)\right] .
\end{aligned}
$$

As in the Dirichlet BC case, the $O(\lambda)$ term in the oneloop approximation is twice the value obtained in conven- 
tional perturbation theory [Eq. (40) with $m=0$ ]. In order to reproduce the latter one has to take into account the two-loop contribution to $\varepsilon$, given by

$$
\widetilde{\varepsilon}^{(2)}=\frac{\lambda}{8}\left[\widetilde{\Delta}_{F}(0)\right]^{2}-\frac{1}{2} M^{2} \widetilde{\Delta}_{F}(0),
$$

where $\widetilde{\Delta}_{F}(x)=\Delta_{F}(x ; m=M)$. Using again results of Sec. III $\mathrm{A}$ and of Appendix $\mathrm{C}$ we obtain

$$
\begin{aligned}
\widetilde{\Delta}_{F}(0) & =\frac{M}{2 \pi^{2} R} F(1 ; M R)+\lim _{\epsilon \rightarrow 0} \frac{\mu^{\epsilon} M^{2-\epsilon} \pi^{(3-\epsilon) / 2}}{\Gamma\left(\frac{1}{2}\right)} \\
& =\frac{1}{R^{2}}\left[\frac{1}{12}-\frac{\lambda^{1 / 2}}{8 \pi \sqrt{6}}+O\left(\frac{\lambda}{\epsilon}\right)\right] .
\end{aligned}
$$

Substituting this into Eq. (46) yields

$$
\tilde{\varepsilon}^{(2)}(R)=\frac{1}{R^{4}}\left[-\frac{\lambda}{1152}+O\left(\frac{\lambda^{2}}{\epsilon}\right)\right] .
$$

Thus, to order $\lambda^{2}$ we finally obtain

$$
\varepsilon(R)=\frac{1}{R^{4}}\left[-\frac{\pi^{2}}{90}+\frac{\lambda}{1152}-\frac{\lambda^{3 / 2}}{288 \sqrt{6} \pi}+O\left(\frac{\lambda^{2}}{\epsilon}\right)\right] .
$$

This agrees to order $\lambda$ with the result found in Sec. III A (in the limit $m \rightarrow 0)$. Besides, we have obtained a correction of order $\lambda^{3 / 2}$. This nonanalyticity in $\lambda$ is a consequence of the fact that the loop expansion in the rearranged Lagrangian is equivalent to a resummation of an infinite number of graphs in the conventional perturbation expansion.

Finally, we note that the UV singularities in the resummed theory depend on $R$, via their dependence on $M$. For instance, in the computation of $\widetilde{\varepsilon}^{(1)}$ a singular term of the form $M^{4} / \epsilon \sim \lambda^{2} / \epsilon R^{4}$ appears in the limit $\epsilon \rightarrow 0$. In the analogous case of TFT it can be shown that the UV singularity present in the one-loop free energy cancels against two- and threeloop contributions in the resummed theory, including a coupling constant renormalization counterterm [12,28]. These contributions on their turn introduce new singularities at $O\left(\lambda^{3}\right)$, which are cancelled by including higher order graphs in the resummed theory, and so on. The situation is exactly the same in our case, so we can safely neglect the $O\left(\lambda^{2}\right)$ term in Eq. (49).

\section{CONCLUSION}

In this paper we have discussed the computation of radiative corrections to the Casimir energy of the massless $\lambda \Phi^{4}$ theory confined between two parallel plates. The case of Dirichlet boundary conditions at the plates was discussed in Sec. II. We obtained an analytical expression for the oneloop self-energy $\Sigma^{(1)}(x)$ both in $D=3$ and $D=4$. The former was shown to be free of IR singularities, in contrast with the claim made in [25].
In the "daisy" resummation of the two-point Green function one is led to solve a Klein-Gordon equation with $\Sigma^{(1)}(x)$ acting as an effective scalar potential. We were able to solve this equation in four dimensions. In spite of $\Sigma^{(1)}$ being negative everywhere, there are no tachyonic modes if the coupling constant $\lambda$ is smaller than $\lambda_{\text {crit }}=8 \pi^{2}$. We then computed the sum of the eigenenergies of the Klein-Gordon operator. Expanding the result in a power series in $\lambda$ one discovers that the $O(\lambda)$ correction does not agree with the result of conventional perturbation theory, and the correction of order $\lambda^{2}$ contains a UV singularity which apparently cannot be renormalized away. The first problem was shown to occur because the sum of zero-point energies does not take into account all the contributions to the vacuum energy in a theory with interaction. As for the second problem, it was argued that the consistent renormalization of the Casimir energy at a given order requires that one takes into account all diagrams to that order. This conjecture is supported by the fact that the Dirichlet BC (in the case of flat boundaries) do not spoil the perturbative renormalizability of the $\lambda \Phi_{4}^{4}$ theory [18].

In the case of periodic boundary conditions in one spatial direction we have argued that the infrared properties of the model are analogous to the one in thermal field theory. In order to define a consistent (i.e., IR finite) perturbative expansion one has to include the screening effects due to collective excitations. A solution to this problem was proposed by Braaten, Pisarski and others in thermal field theory [1012]. It consists in the resummation of an infinite class of diagrams, which gives the field an effective mass. This can be done in a systematic way using the Braaten-Pisarski resummation method. This was illustrated with the calculation of the leading and next-to-leading order radiative corrections to the Casimir energy. Besides, our calculation shows that the resummed weak coupling expansion of the Casimir energy in the case of periodic $\mathrm{BC}$ contains fractional powers of $\lambda$, in contrast to the case of Dirichlet boundary conditions.

We note that calculations of radiative corrections to the Casimir energy via the resummation of zero-point energies have appeared recently in the literature [29], without paying due attention to the subtleties of the resummed perturbation theory. As we have shown, this may lead to inconsistencies in the results [30].

Finally, we hope that the techniques discussed here may be useful in investigations of Kaluza-Klein compactification scenarios, as well as in the study of surface critical phenomena.

\section{ACKNOWLEDGMENTS}

The authors acknowledge the financial support from FAPESP under grants 00/03277-3 (L.C.A.) and 98/11646-7 (R.M.C.), and from FAPERJ (R.M.C.). They also acknowledge the kind hospitality of the Departamento de Física Matemática, Universidade de São Paulo, where this work was initiated. 


\section{APPENDIX A}

We discuss the solution to the equation

$$
\left(\frac{d^{2}}{d y^{2}}+k^{2}+\frac{g}{\sin ^{2} y}\right) \varphi(y)=0
$$

with Dirichlet boundary conditions at $y=0$ and $y=\pi$.

Let us first consider the asymptotic behavior of its solutions near one of the boundaries (say, at $y=0$ ). To this end, we can replace Eq. (A1) by

$$
\left(\frac{d^{2}}{d y^{2}}+\frac{g}{y^{2}}\right) \varphi(y)=0 .
$$

The most general solution to Eq. (A2) is

$$
\varphi(y)=A y^{s_{+}}+B y^{s_{-}},
$$

where $s_{ \pm} \equiv \frac{1}{2}(1 \pm \sqrt{1-4 g})$. If $g<1 / 4$, the boundary condition $\varphi(0)=0$ is not enough to fix the relation between $A$ and $B$, as both $y^{s_{+}}$and $y^{s_{-}}$vanish at $y=0$. To resolve this indeterminacy, we follow [31] and regularize the potential near the origin: $V_{R}(y)=-g / y^{2}$ for $y>a$, and $V_{R}(y)=-g / a^{2}$ for $y<a$. At the end, we shall take the limit $a \rightarrow 0$.

For $y>a$, the solution is given by Eq. (A3). For $y<a$, the solution which satisfies the boundary condition at the origin is $\varphi(y)=C \sin (\sqrt{g} y / a)$. Continuity of $\varphi(y)$ and its derivative at $y=a$ implies the relation $B / A \sim a^{s_{+}{ }^{-s_{-}}}$as $a \rightarrow 0$, i.e., only the solution with the faster decay at the origin survives when the regularization is removed. If $g>1 / 4, s_{+}-s_{-}$is purely imaginary and $\lim _{a \rightarrow 0} B / A$ does not exist. This sets a critical value to $g$, namely $g_{\text {crit }}=1 / 4$, above which the "Hamiltonian" $H=-d^{2} / d y^{2}-g / y^{2}$ is unbounded from below [31].

Let us return to the complete equation (A1). It is convenient to make some changes of variables. First, we set $y$ $=i x+\pi / 2$ and define $s \equiv \frac{1}{2}(-1+\sqrt{1-4 g})$ [so that $s(s$ $+1)=-g]$. This transforms Eq. (A1) into

$$
\left[-\frac{d^{2}}{d x^{2}}+k^{2}-\frac{s(s+1)}{\cosh ^{2} x}\right] \varphi(x)=0 .
$$

Then, we make $\xi=\tanh x$ and obtain

$$
\frac{d}{d \xi}\left[\left(1-\xi^{2}\right) \frac{d \psi}{d \xi}\right]+\left[s(s+1)-\frac{k^{2}}{1-\xi^{2}}\right] \varphi(\xi)=0 .
$$

Finally, we put $\varphi(\xi)=\left(1-\xi^{2}\right)^{k / 2} w(\xi)$, followed by the change of variable $\xi=2 u-1$, to get

$$
\begin{aligned}
& u(1-u) \frac{d^{2} w}{d u^{2}}+[1+k-2(1+k) u] \frac{d w}{d u}-(k-s)(k+s+1) w \\
& =0 .
\end{aligned}
$$

Equation (A6) is the hypergeometric differential equation [24] with parameters $\alpha=k-s, \beta=k+s+1$, and $\gamma=1+k$. Its general solution may be written as

$$
\begin{aligned}
w(u)= & A(1-u)^{-k} F(-s, s+1 ; 1+k ; u) \\
& +B u^{-k} F(-s, s+1 ; 1-k ; u),
\end{aligned}
$$

where $F(\alpha, \beta ; \gamma ; z)$ is the hypergeometric function. Returning to the variable $y$ and the function $\varphi(y)$, we have

$$
\begin{aligned}
\varphi(y)= & A^{\prime} e^{-i k y} F\left(-s, s+1 ; 1+k ; \frac{i e^{-i y}}{2 \sin y}\right) \\
& +B^{\prime} e^{i k y} F\left(-s, s+1 ; 1-k ; \frac{i e^{-i y}}{2 \sin y}\right) .
\end{aligned}
$$

The asymptotic behavior of $\varphi(y)$ as $y \rightarrow 0$ may be extracted from $\lim _{z \rightarrow 0} F(\alpha, \beta ; \gamma ; z)=1$, after using the relation [valid for $|\arg (-z)|<\pi,|\arg (1-z)|<\pi, \quad \alpha-\beta \neq 0, \pm 1$, $\pm 2, \ldots]$

$F(\alpha, \beta ; \gamma ; z)$

$$
\begin{aligned}
= & (-z)^{-\alpha} \frac{\Gamma(\gamma) \Gamma(\beta-\alpha)}{\Gamma(\gamma-\alpha) \Gamma(\beta)} F\left(\alpha, 1+\alpha-\gamma ; 1+\alpha-\beta ; \frac{1}{z}\right) \\
& +(-z)^{-\beta} \frac{\Gamma(\gamma) \Gamma(\alpha-\beta)}{\Gamma(\gamma-\beta) \Gamma(\alpha)} F\left(\beta, 1+\beta-\gamma ; 1+\beta-\alpha ; \frac{1}{z}\right) .
\end{aligned}
$$

In this way,

$$
\begin{aligned}
\varphi(y) \stackrel{y \rightarrow}{\sim} & A^{\prime} F\left(-s, s+1 ; 1+k ; \frac{i}{2 y}\right) \\
& +B^{\prime} F\left(-s, s+1 ; 1-k ; \frac{i}{2 y}\right) \\
\sim & {\left[A^{\prime} \frac{\Gamma(1+k)}{\Gamma(1+k+s)}+B^{\prime} \frac{\Gamma(1-k)}{\Gamma(1-k+s)}\right] \frac{\Gamma(2 s+1)}{\Gamma(s+1)} } \\
& \times\left(-\frac{i}{2 y}\right)^{s}+\left[A^{\prime} \frac{\Gamma(1+k)}{\Gamma(k-s)}+B^{\prime} \frac{\Gamma(1-k)}{\Gamma(-k-s)}\right] \\
& \times \frac{\Gamma(-2 s-1)}{\Gamma(-s)}\left(-\frac{i}{2 y}\right)^{-s-1} .
\end{aligned}
$$

Recalling the analysis of Eq. (A2), we impose that $\varphi(y)$ $\sim y^{s+1}$ as $y \rightarrow 0$. This can be accomplished by taking $B^{\prime}$ $=0$ and $k=-(n+s)(n=1,2, \ldots)$, that is

$$
\varphi(y)=A^{\prime} e^{i(n+s) y} F\left(-s, s+1 ;-s-n+1 ; \frac{i e^{-i y}}{2 \sin y}\right) .
$$

The boundary condition at $y=\pi$ is also satisfied by this solution, since $\varphi(y) \sim(\pi-y)^{s+1}$ as $y \rightarrow \pi$. Hence, Eq. (A11) is an acceptable solution to Eq. (A1). [Remark: we could also have taken $A^{\prime}=0$ and $k=n+s \quad(n=1,2, \ldots)$ in Eq. (A10), but this leads to the same values of $k^{2}$ and to the same solutions given by Eq. (A11).] 


\section{APPENDIX B}

The goal here is to obtain the analytical continuation of the function $\mathcal{H}\left(z ; s, a^{2}\right)$, defined in Eq. (22), to the whole complex $z$ plane. To this end, we shall use the Plana summation formula [32]

$$
\sum_{k=M}^{N} f(k)=\frac{1}{2}[f(M)+f(N)]+\int_{M}^{N} f(x) d x-i \int_{0}^{\infty} d y \frac{f(N+i y)-f(M+i y)-f(N-i y)+f(M-i y)}{e^{2 \pi y}-1} .
$$

In the present case, we choose $M=1, N=\infty$, and $f(x)$ $=\left[(x+s)^{2}+a^{2}\right]^{-z}$. In order to apply the Plana formula some conditions have to be satisfied [32]. First, we assume that $\mathfrak{R} z>\frac{1}{2}$, so that the series in Eq. (22) converges absolutely. Then, it can be shown that the function $f(\tau+i t)$ is holomorphic for $\tau \geqslant 1$ for any $t$, and that $\lim _{t \rightarrow \pm \infty} e^{-2 \pi|t|} f(\tau+i t)=0$ uniformly in the interval $1 \leqslant \tau$ $<\infty$. In addition, $\lim _{\tau \rightarrow \infty} \int_{-\infty}^{\infty} d t e^{-2 \pi|t|}|f(\tau+i t)|=0$. Under these conditions, we have

$$
\begin{aligned}
\mathcal{H}\left(z ; s, a^{2}\right)= & \frac{1}{2}\left[(s+1)^{2}+a^{2}\right]^{-z}+\int_{1}^{\infty} \frac{d x}{\left[(x+s)^{2}+a^{2}\right]^{z}} \\
& +i \int_{0}^{\infty} d t \frac{f(1+i t)-f(1-i t)}{e^{2 \pi t}-1} .
\end{aligned}
$$

The first integral can be computed in closed form:

$$
\begin{aligned}
& \int_{1}^{\infty} \frac{d x}{\left[(x+s)^{2}+a^{2}\right]^{z}} \\
& \quad=\frac{(1+s)^{1-2 z}}{2 z-1} F\left(z, z-\frac{1}{2} ; z+\frac{1}{2} ;-\left(\frac{a}{1+s}\right)^{2}\right) .
\end{aligned}
$$

Equations (B2) and (B3) give the analytic continuation of $\mathcal{H}\left(z ; s, a^{2}\right)$ to the whole complex $z$-plane. From the definition of the hypergeometric function it follows that $\mathcal{H}\left(z ; s, a^{2}\right)$ has simple poles at $z=1 / 2,-1 / 2,-3 / 2, \ldots$.

\section{APPENDIX C}

Consider the function

$$
\Psi_{\epsilon}(\alpha) \equiv \frac{\mu^{\epsilon}}{2 R} \sum_{n=-\infty}^{\infty} \int \frac{d^{d-\epsilon} p}{(2 \pi)^{d-\epsilon}}\left[p^{2}+\left(\frac{2 \pi n}{R}\right)^{2}+m^{2}\right]^{\alpha}
$$

Integration over $p$ leads to

$$
\Psi_{\epsilon}(\alpha)=\frac{\mu^{\epsilon}}{2^{d+1-\epsilon} R} \frac{\pi^{\alpha}}{\Gamma(-\alpha)} S\left(m, \frac{R}{2} ;-2 \alpha-d+\epsilon\right),
$$

$$
S(m, a ; s) \equiv \pi^{-s / 2} \Gamma\left(\frac{s}{2}\right) \sum_{n=-\infty}^{\infty}\left[\left(\frac{m}{\pi}\right)^{2}+\left(\frac{n}{a}\right)^{2}\right]^{-s / 2} .
$$

The series converges absolutely for $\mathfrak{R}>>1$. The analytical continuation to a meromorphic function in the complex $s$ plane is given by [33]

$S(m, a ; s)$

$$
=\frac{a m^{1-s}}{\pi^{(1-s) / 2}}\left[\Gamma\left(\frac{s-1}{2}\right)+4(m a)^{(s-1) / 2} F\left(\frac{1-s}{2} ; 2 m a\right)\right],
$$

where

$$
F(s ; a) \equiv \sum_{n=1}^{\infty} n^{-s} K_{s}(n a)
$$

with $K_{s}(x)$ the modified Bessel function of the second kind. The following expansions, valid for $a \ll 1$, will be useful [34]:

$$
\begin{aligned}
& F(1 ; a)=\frac{\pi^{2}}{6 a}-\frac{\pi}{2}+O(a \ln a), \\
& F(2 ; a)=\frac{\pi^{4}}{45 a^{2}}-\frac{\pi^{2}}{12}+\frac{\pi a}{6}+O\left(a^{2}\right) .
\end{aligned}
$$

Using the analytical continuation given in Eq. (C4), one may write $\Psi_{\epsilon}(\alpha)$ (in the limit $\epsilon \rightarrow 0$ ) as the sum of two terms, namely, $\lim _{\epsilon \rightarrow 0} \Psi_{\epsilon}(\alpha)=\mathcal{A}(\alpha)+\mathcal{B}(\alpha)$, where

$$
\mathcal{A}(\alpha)=\frac{2^{(1+2 \alpha-d) / 2}}{\pi^{(1+d) / 2} \Gamma(-\alpha)}\left(\frac{m}{R}\right) \stackrel{(1+2 \alpha+d) / 2}{ } F\left(\frac{1+2 \alpha+d}{2} ; m R\right)
$$

and

$$
\mathcal{B}(\alpha)=\lim _{\epsilon \rightarrow 0} \frac{\mu^{\epsilon} m^{1+2 \alpha+d-\epsilon}}{2^{d+2-\epsilon} \pi^{(1+d-\epsilon) / 2}} \frac{\Gamma\left(\frac{-1-2 \alpha-d+\epsilon}{2}\right)}{\Gamma(-\alpha)} .
$$

where

Note that only $\mathcal{A}(\alpha)$ depends on $R$. 
[1] S. A. Fulling, Aspects of Quantum Field Theory in Curved Space-Time (Cambridge University Press, Cambridge, England, 1989).

[2] Modern Kaluza-Klein Theories, edited by T. Appelquist, A. Chodos, and P. G. O. Freund (Addison-Wesley, Menlo Park, CA, 1987).

[3] G. Plunien, B. Muller, and W. Greiner, Phys. Rep. 134, 87 (1986); V. M. Mostepanenko and N. N. Trunov, Sov. Phys. Usp. 31, 965 (1988); M. Bordag, U. Mohideen, and V. M. Mostepanenko, Phys. Rep. 353, 1 (2001).

[4] A. Chodos, R. L. Jaffe, K. Johnson, C. B. Thorn, and V. F. Weisskopf, Phys. Rev. D 9, 3471 (1974).

[5] J. I. Kapusta, Finite Temperature Field Theory (Cambridge University Press, Cambridge, England, 1989); M. Le Bellac, Thermal Field Theory (Cambridge University Press, Cambridge, England, 1996).

[6] H. W. Diehl, in Phase Transitions and Critical Phenomena, edited by C. Domb and J. L. Lebowitz (Academic Press, London, 1986), Vol. 10.

[7] L. H. Ford, Proc. R. Soc. London A368, 305 (1979); L. H. Ford and T. Yoshimura, Phys. Lett. 70A, 89 (1979).

[8] D. J. Toms, Phys. Rev. D 21, 928 (1980); 21, 2805 (1980); Ann. Phys. (N.Y.) 129, 334 (1980).

[9] G. Denardo and E. Spallucci, Nucl. Phys. B169, 514 (1980); A. Actor, Class. Quantum Grav. 7, 663 (1990); 7, 1463 (1990); L. H. Ford and N. F. Svaiter, Phys. Rev. D 51, 6981 (1995).

[10] E. Braaten and R. D. Pisarski, Nucl. Phys. B337, 569 (1990).

[11] R. R. Parwani, Phys. Rev. D 45, 4695 (1992); 48, 5965(E) (1993).

[12] J. Frenkel, A. V. Saa, and J. C. Taylor, Phys. Rev. D 46, 3670 (1992).

[13] M. R. Douglas and N. A. Nekrasov, hep-th/0106048.

[14] W. Selke, in Phase Transitions and Critical Phenomena, edited by C. Domb and J. L. Lebowitz (Academic Press, London, 1992), Vol. 15.

[15] Y. Hosotani, Phys. Lett. B 319, 332 (1993); Phys. Rev. D 51, 2022 (1995).

[16] M. Bordag and D. Robaschik, Ann. Phys. (N.Y.) 165, 192
(1985); E. Wieczorek, D. Robaschik, and K. Scharnhorst, Sov. J. Nucl. Phys. 44, 665 (1986); D. Robaschik, K. Scharnhorst, and E. Wieczorek, Ann. Phys. (N.Y.) 174, 401 (1987); M. Bordag and K. Scharnhorst, Phys. Rev. Lett. 81, 3815 (1998); M. Bordag and J. Lindig, Phys. Rev. D 58, 045003 (1998); F. Ravndal and J. B. Thomassen, ibid. 63, 113007 (2001); K. Melnikov, ibid. 64, 045002 (2001).

[17] C. M. Bender and P. Hays, Phys. Rev. D 14, 2622 (1976); K. A. Milton, ibid. 22, 1441 (1980); 25, 3441(E) (1982).

[18] K. Symanzik, Nucl. Phys. B190, 1 (1981).

[19] A. A. Actor and I. Bender, Phys. Rev. D 52, 3581 (1995).

[20] L. C. de Alburquerque, Phys. Rev. D 55, 7754 (1997).

[21] L. H. Ford and N. F. Svaiter, Phys. Rev. D 58, 065007 (1998).

[22] R. Balian and B. Duplantier, Ann. Phys. (N.Y.) 112, 165 (1978).

[23] N. N. Bogoliubov and D. V. Shirkov, Introduction to the Theory of Quantized Fields (Interscience, New York, 1959).

[24] I. S. Gradshteyn and I. M. Ryzhik, Tables of Integrals, Series, and Products (Academic Press, New York, 1994).

[25] C. D. Fosco and N. F. Svaiter, hep-th/9910068.

[26] An alternative form of the analytical continuation of $\mathcal{H}\left(z ; s, a^{2}\right)$ can be found in the book by E. Elizalde, S. D. Odintsov, A. Romeo, A. A. Bytsenko, and S. Zerbini, Zeta Regularization Techniques with Applications (World Scientific, Singapore, 1994).

[27] S. Coleman and E. Weinberg, Phys. Rev. D 7, 1888 (1973).

[28] R. Parwani and H. Singh, Phys. Rev. D 51, 4518 (1995).

[29] S. Nam, J. High Energy Phys. 10, 044 (2000); W. H. Huang, Phys. Lett. B 497, 317 (2001); A. A. Bytsenko, A. E. Gonçalves, and S. Zerbini, Mod. Phys. Lett. A 16, 1479 (2001).

[30] L. C. de Albuquerque and R. M. Cavalcanti, J. High Energy Phys. 07, 025 (2001).

[31] L. D. Landau and E. M. Lifshitz, Quantum Mechanics (Pergamon, Oxford, 1985).

[32] E. T. Whittaker and G. N. Watson, A Course of Modern Analysis (Cambridge University Press, Cambridge, England, 1996).

[33] J. Ambjørn and S. Wolfram, Ann. Phys. (N.Y.) 147, 1 (1983).

[34] H. W. Braden, Phys. Rev. D 25, 1028 (1982). 